\title{
The Contribution of Symptoms of Posttraumatic Stress Disorder, Health Anxiety and Intolerance of Uncertainty to Distress in Ménière Disease
}

\author{
Sarah E. Kirby, PhD, and Lucy Yardley, PhD
}

\begin{abstract}
This study assessed whether symptoms of posttraumatic stress disorder (PTSD), health anxiety, and intolerance of uncertainty were associated with distress in members of the Ménière Society $(n=800)$, and compared the extent of anxiety, depression, intolerance of uncertainty, and health anxiety with a healthy control group $(n=484)$. PTSD symptoms were associated with anxiety, depression, and handicap. Health anxiety was associated with anxiety and depression. Intolerance of uncertainty was directly associated with anxiety; its association with depression and handicap was mediated by PTSD symptoms. The Ménière group reported more anxiety, depression, and health anxiety than the control group, but were not more intolerant of uncertainty. More than half of the Ménière group reported experiencing partial or full PTSD symptoms. As PTSD, health anxiety and intolerance of uncertainty are modifiable with psychological treatment, we advise that clinicians should screen patients with Ménière disease who are particularly distressed.
\end{abstract}

Key Words: Vertigo, vestibular disease, dizziness, psychological adjustment, quality of life.

( $J$ Nerv Ment Dis 2009;197: 000-000)

$\mathrm{M}$ énière disease is a chronic, incurable disease that progressively damages the parts of the inner ear responsible for balance and hearing. Symptoms comprise of intermittent, unpredictable attacks of severe vertigo (a strong sense of spinning and unsteadiness, sweating, nausea, and vomiting), tinnitus, a sensation of pressure or fullness in the ear, and progressive hearing loss that becomes permanent in 1 or in both the ears. These symptoms have a negative impact on quality of life (Yardley et al., 2003), and high levels of distress are often reported among people with Ménière disease (Best et al., 2006; Cohen et al., 1995). The relationship between Ménière disease and distress is a complex one, as the vestibular and autonomic systems are neurologically linked (Balaban and Thayer, 2001; Brandt, 2000; Furman and Jacob, 2001). Autonomic arousal can be triggered by vestibular disturbance as well as anxiety arousal, leading to the inclusion of vestibular disorder as an example in the DSM-IV-TR criterion for anxiety disorder due to a general medical condition.

The presence of an anxiety disorder can have a negative impact on quality of life (Cramer et al., 2005), however, few psychological interventions have been developed to attempt to reduce distress in people with Ménière disease. Cognitive behavioral therapy (CBT) has been used successfully to reduce distress in many

AQ:6 School of Psychology, University of Southampton, Southampton, United Kingdom. Supported by the Ménière Society, United Kingdom.

The contribution of symptoms of posttraumatic stress disorder (PTSD), health anxiety and intolerance of uncertainty to distress in Ménière disease.

Send reprint requests to Sarah Kirby, PhD, School of Psychology, University of Southampton, Highfield, Southampton, SO17 1BJ, United Kingdom. E-mail: sek@soton.ac.uk.

Copyright (C) 2009 by Lippincott Williams \& Wilkins

ISSN: 0022-3018/09/19705-0001

DOI: 10.1097/NMD.0b013e3181a20866 chronic disorders, and case studies have suggested that CBT may be useful for people with Ménière disease (Elwood et al., 1982; Hagnebo et al., 1998). Elements of CBT have been incorporated into physical rehabilitation programs for chronic dizziness and brief self-help interventions for Ménière disease (Andersson et al., 2006; Jacob et al., 2001; Johansson et al., 2001; Yardley and Kirby, 2006). These interventions have achieved reduction in symptoms but have had only limited effects on anxiety and depression. To develop more extensive psychological interventions for people with Ménière disease, we need to understand the modifiable psychological processes and mechanisms involved.

A systematic review (Kirby and Yardley, 2008) identified posttraumatic stress disorder (PTSD) and health anxiety as 2 possible mechanisms that may contribute to distress in Ménière disease, but their potential role needs to be tested empirically. PTSD is diagnosed when a traumatic event is experienced with a sense of fear, helplessness or horror, and results in distress or impairment in functioning. The addition of "being diagnosed with a life threatening illness" as a possible traumatic event in the DSM-IV criterion for PTSD has led to the reporting of PTSD following a range of medical illnesses and treatments (Kangas et al., 2002; Tedstone and Tarrier, 2003). In Ménière disease, attacks of vertigo have been described as terrifying, resulting in feelings of helplessness and powerlessness (Yardley, 1997). The first attack in particular may be perceived as life threatening, as people do not know what is happening to them (Pollak et al., 2003; Yardley, 1997). Symptoms of PTSD such as avoidance behavior and increased levels of arousal have been reported among people with vestibular disorders (Cohen et al., 1995; Jacob et al., 2002; Yardley et al., 1998). Vertigo attacks are recurrent, and PTSD can result from cumulative events (Alonzo, 2000). Even when people do not fulfil the full DSM diagnostic criteria, they may still suffer from some PTSD symptoms which need treatment (Mylle and Maes, 2004). It seems likely that some people with Ménière disease may develop some or all of the features of PTSD.

Health anxiety was defined by Fava et al., (1995) as a generic worry about illness and preoccupation with somatic sensations. Furer et al. (2001) have suggested that people have an increased risk of developing health anxiety if they have a condition that is chronic, intermittent, degenerative, involves uncertainty, or has no clearly recognized cause. As all these risk factors are features of Ménière disease, people with Ménière disease may be vulnerable to developing health anxiety. Indeed, a recent study (Best et al., 2006) found that people with Ménière disease had higher rates of anxiety, depression, and somatoform disorders than those with other types of vestibular disorder (such as BPPV and vestibular neuritis).

In addition to PTSD and health anxiety, this study also investigates the possible contribution of a third mechanism to distress, intolerance of uncertainty. Intolerance of uncertainty is a cognitive bias whereby a person will evaluate uncertain situations as negative and stressful, and believe that uncertain situations should be avoided (Dugas et al., 2005). Dislike of the uncertainty of attacks among people who experience chronic vertigo is often noted anecdotally (Yardley, 1997). In Ménière disease, attacks are particularly 
unpredictable, varying greatly in severity and duration, with periods of remission between attacks ranging from days to years. The first study to investigate the impact of intolerance of uncertainty on distress in people with Ménière disease found that intolerance of uncertainty was indeed strongly associated with anxiety among people with Ménière disease (Kirby and Yardley, 2009). It has been suggested that intolerance of uncertainty may be an underpinning feature of anxiety disorders in general (Holaway et al., 2006), with people with anxiety disorders having higher levels of intolerance of uncertainty than nonclinical controls (Ladouceur et al., 1999). The purpose for including intolerance of uncertainty in this study, therefore, was to see whether the findings of the previous study could be replicated, and to assess whether intolerance of uncertainty contributes independently to distress in people with Ménière disease when included with health anxiety and PTSD. Like PTSD and health anxiety, intolerance of uncertainty has been treated successfully in other populations using psychological interventions (Dugas and Ladouceur, 2000; Jones, 2002; National Institute for Clinical Excellence, 2005).

The primary aim of this study was to assess whether PTSD, health anxiety, and intolerance of uncertainty are associated with distress (i.e., anxiety, depression, and handicap) in people with Ménière disease, after controlling for demographic and illness characteristics. A secondary aim was to compare the extent of anxiety, depression, intolerance of uncertainty, and health anxiety in people with Ménière disease group and a healthy control group.

\section{METHOD}

\section{Participant Recruitment and Procedure}

Participants were recruited by sending questionnaire packs to 1400 randomly selected members of the Ménière Society, inviting them to participate. Members were asked to return the pack if they did not want to take part in the study. Up to 2 reminders were sent to nonresponders at 1 month intervals. To be eligible to participate, participants were required to indicate that they had received a diagnosis of Ménière disease from their doctor. Control participants were recruited by including a control questionnaire in the initial pack sent to the members, to be completed by a willing friend or relative without Ménière disease or severe dizziness. The study was approved by the Ethical Committee of the School of Psychology, University of Southampton.

\section{Measures}

Single items were used to assess age, gender, length of time since symptoms began (illness duration), and length of time since last attack (illness recency). Vertigo and somatic anxiety were assessed using the long version of the Vertigo Symptom Scale (Yardley et al., 1992). Tinnitus and fullness in the ear were assessed using the Tinnitus Severity Index and the Aural Pressure Index (Cass, 1999; Stahle et al., 1981). Hearing disability was assessed using the Hearing Disability Questionnaire (Lutman et al., 1987).

The English version of the Intolerance of Uncertainty Scale (Buhr and Dugas, 2002) was used to assess how participants might interpret and respond to uncertainty. Health anxiety was measured using the short-form Health Anxiety Inventory (Salkovskis et al., 2002). Although the short-form Health Anxiety Inventory is suitable for use with people who have a physical illness, minor amendments were made to the items that refer to having a serious disease, to specify a serious disease other than Ménière disease. To assess clinical levels of health anxiety, scores were recoded to show no health anxiety if scores were in the 0 to 14 range, high health anxiety if scores were in the 15 to 17 range, and hypochondriasis if scores were 18 or more (Rode et al., 2006).

PTSD was measured using the PTSD checklist (PCL) (Weathers et al., 1993), a 17-item scale that follows the DSM-IV criteria for PTSD. For the purposes of this study, the introductory text and questions were worded to specifically refer to the extent that respondents had experienced symptoms in the past month in relation to a severe Ménière attack. Two different methods were used to determine clinical levels of PTSD. The first method was proposed by Blanchard et al. (1996) who recommended that a simple score of 44 or more is used to identify PTSD. The second method was proposed by the National Center for PTSD who recommended symptomatic clusters are required in addition to a total score of 44 or more (Weathers et al., 1993). The symptomatic cluster requirements for clinical levels of criterion B (re-experiencing) are that at least 1 item on that subscale must be scored 3 or above. The requirements for clinical levels of criterion $\mathrm{C}$ (avoidance/numbing) are that at least 3 items on that subscale must be scored 3 or above. To meet the requirement for clinical levels of criterion D (arousal), at least 2 items on that subscale must be scored 3 or above.

Depression and anxiety were measured using the Hospital Anxiety and Depression Scale (Zigmond and Snaith, 1983). To determine clinical levels, anxiety and depression were coded as non clinical if scores were in the 0 to 7 range, possibly clinical if scores were in the 8 to 10 range, and clinical if scores were 11 or more (Zigmond and Snaith, 1983). Handicap resulting from dizziness was measured using the total score of the Dizziness Handicap Inventory (Jacobson and Newman, 1990).

\section{Statistical Analysis}

Hierarchical regression was used to assess the effects of the psychological variables on distress, after controlling for demographic and illness characteristics. Age and gender were entered into the first step; illness characteristics were entered into the second step; and PTSD, health anxiety, and intolerance of uncertainty were entered on the final step. These regressions were carried out to identify variables associated with anxiety, depression, and handicap. Post hoc regression analyses were carried out to test whether PTSD symptoms mediated the relationship of intolerance of uncertainty to depression and handicap (Baron and Kenny, 1986). To test whether these reductions in effect were significant, the Aroian version of the Sobel test was used (Baron and Kenny, 1986).

MANOVA was used to compare the extent of anxiety, depression, intolerance of uncertainty, and health anxiety in people with Ménière disease and the healthy control group. Although preliminary $t$-tests found age and gender differences between the groups, MANCOVA could not be used to control for age and gender due to violation of the homogeneity of regression assumption. Therefore, age was dichotomized ( $<60$ vs. $\geq 60$ years) and included with gender and group (Ménière disease vs. control) as fixed factors to test for any interactions between these variables. Follow-up ANOVAs were carried out to identify which dependent variables the Ménière disease and control group differed on.

\section{RESULTS}

Of the 1400 members of the Ménière Society who were invited to take part in the study, data from $800(57.1 \%)$ were included in the final analyses (mentioned in Fig. 1 for reasons for nonparticipation or exclusion). The age range was 25 to 90 years, with $295(36.9 \%)$ men and 505 (63.1\%) women. In the control group, data from 484 (34.6\%) were included in the analyses. The control group comprised 216 (44.6\%) men and 268 (55.4\%) women, and the age range was 18 to 93 years. The means and standard deviations for all variables measured in both groups are presented in Table 1.

\section{Variables Associated With Distress}

The bivariate correlations between demographic and illness characteristics, psychological variables, and the 3 measures of dis- 


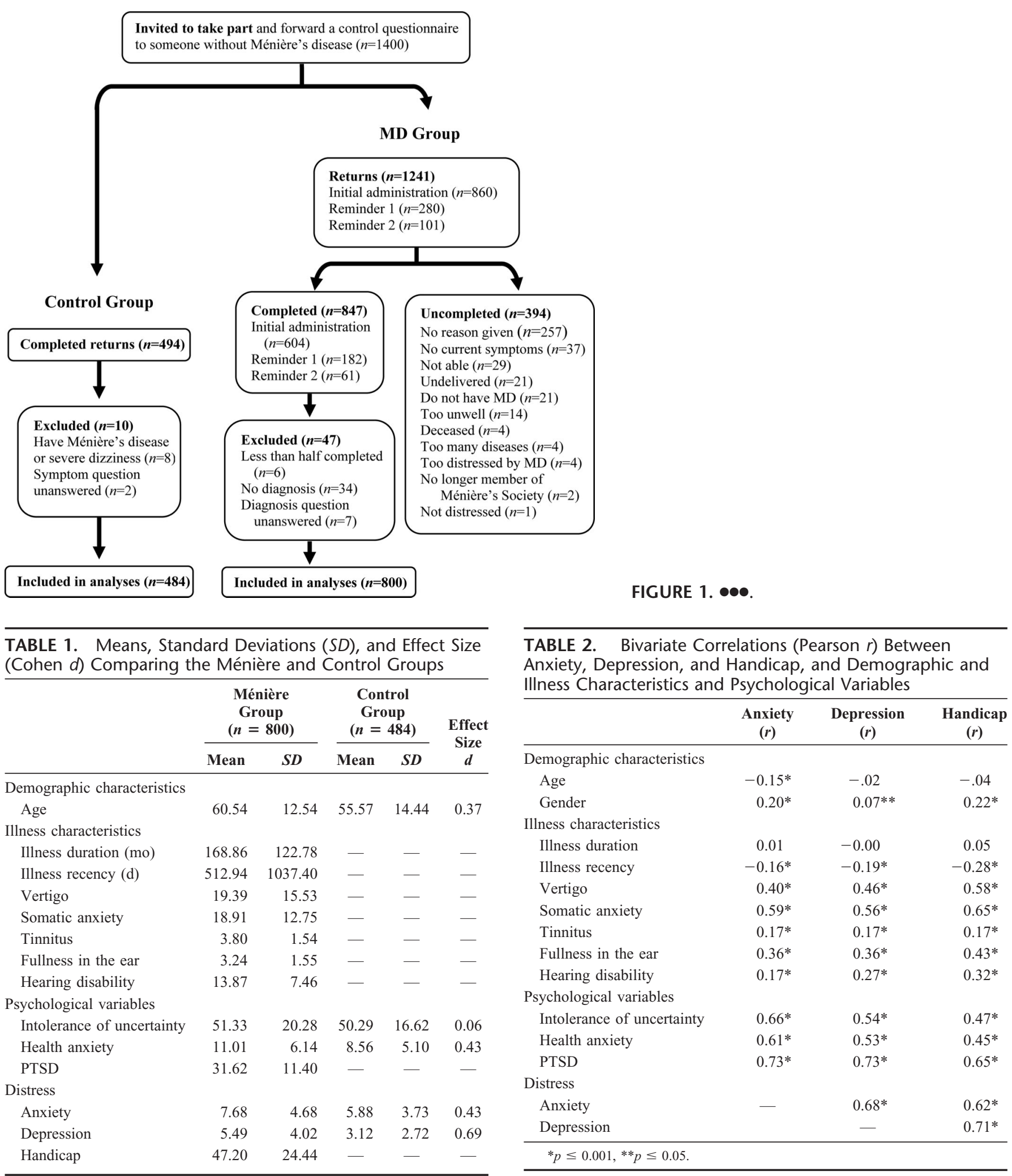

tress (anxiety, depression, and handicap) are shown in Table 2. With the exception of illness duration and age (for depression and handicap), all variables were significantly correlated with anxiety, de- pression, and handicap. Large effect sizes were observed for the presence of PTSD and health anxiety symptoms, intolerance of uncertainty, and reporting more somatic anxiety and vertigo. 
TABLE 3. Beta Coefficients for the Final Regression Models for Anxiety, Depression, and Handicap, Controlling for Demographic Variables and Symptom Severity

\begin{tabular}{lccc}
\hline & $\begin{array}{c}\text { Anxiety } \\
(\boldsymbol{\beta})\end{array}$ & $\begin{array}{c}\text { Depression } \\
(\boldsymbol{\beta})\end{array}$ & $\begin{array}{c}\text { Handicap } \\
(\boldsymbol{\beta})\end{array}$ \\
\hline Demographic characteristics & & & \\
Age & -0.04 & $0.10^{*}$ & $0.07^{* *}$ \\
Gender & $0.09^{*}$ & -0.02 & $0.11^{*}$ \\
Illness characteristics & & & \\
Illness duration & 0.02 & -0.03 & 0.04 \\
Illness recency & 0.02 & 0.00 & $-0.08^{*}$ \\
Vertigo & 0.01 & $0.14^{*}$ & $0.24^{*}$ \\
Somatic anxiety & $0.18^{*}$ & $0.11^{* *}$ & $0.20^{*}$ \\
Tinnitus & -0.01 & 0.00 & -0.03 \\
Fullness in the ear & 0.02 & 0.02 & $0.07^{* *}$ \\
Hearing disability & 0.00 & $0.07^{* *}$ & $0.14^{*}$ \\
Psychological variables & & & \\
Intolerance of uncertainty & $0.24^{*}$ & $0.06^{* * *}$ & $0.07^{* * *}$ \\
Health anxiety & $0.17^{*}$ & $0.15^{*}$ & $0.07^{* * *}$ \\
PTSD & $0.35^{*}$ & $0.45^{*}$ & $0.27^{*}$ \\
\hline${ }^{*} p \leq 0.001, * * p \leq 0.01, * * * p \leq 0.05$. & & \\
\hline
\end{tabular}

Beta coefficients for the final regression models for anxiety, depression, and handicap are shown in Table 3 . For anxiety, $66 \%$ of the variance was accounted for by the final regression equation $(F(12,787)=124.48, p<0.001)$. Illness characteristics accounted for $31 \%$ of this variance $\left(F R^{2}\right.$ change $\left.(7790)=56.07, p<0.001\right)$, and the psychological variables accounted for a further $28 \%\left(F R^{2}\right.$ change $(3787)=215.485, p<0.001)$. In the final model, the variables that contributed most to anxiety were the presence of PTSD symptoms, and intolerance of uncertainty. Experiencing symptoms of somatic anxiety and reporting more health anxiety made the next largest contributions to anxiety.

A total of $59 \%$ of the variance in depression was accounted for by the final regression equation $(F(12,787)=95.28, p<0.001)$. Illness characteristics accounted for $36 \%$ of the variance $\left(F R^{2}\right.$ change $(7790)=64.79, p<0.001)$, and psychological variables accounted for a further $22 \%\left(F R^{2}\right.$ change $(3787)=144.34, p<$ $0.001)$. The variables that contributed most to the final model of depression were the presence of PTSD symptoms, health anxiety, and vertigo symptoms.

The final regression equation accounted for $63 \%$ of the variance for handicap $(F(12,787)=110.43, p<0.001)$. Most of the variance $(49 \%)$ was accounted for by illness characteristics $\left(F R^{2}\right.$ change $(7790)=121.85, p<0.001)$, with only a further $9 \%$ of the variance being accounted for by the psychological variables $\left(F R^{2}\right.$ change $(3787)=60.01, p<0.001)$. In the final model, PTSD symptoms contributed most to handicap, followed by symptoms of vertigo, somatic anxiety, and hearing disability.

PTSD almost fully mediated the relationship of intolerance of uncertainty with handicap and depression. The inclusion of PTSD reduced the association between intolerance of uncertainty and depression from a standardized Beta coefficient of 0.54 to 0.08 , which was only just still significant $(p=0.022)$. For handicap, the inclusion of PTSD as a mediator reduced the strength of association with intolerance of uncertainty from a standardized Beta coefficient of 0.48 to 0.05 , which was no longer significant ( $p=0.138$ ). The standardized Beta coefficient for the relationship between intolerance of uncertainty and PTSD was $0.68(p<0.001)$. The results suggest that PTSD significantly mediated the effect of intolerance of uncertainty on depression (Aroian $=15.61, p<0.001)$ and handicap $($ Aroian $=14.12, p<0.001)$.

\section{Comparison Between Ménière Disease and Control Groups}

The secondary aim of this study was to compare the extent of anxiety, depression, intolerance of uncertainty, and health anxiety in people with Ménière disease group and a healthy control group (mentioned in Table 1 for means). Significant differences were found between the Ménière disease and control group (Wilks' $\Lambda=0.88, F(4$, $1273)=43.03, p<0.001, \eta_{p}^{2}=0.12$ ). Participants in the Ménière disease group were significantly more anxious and depressed than controls (anxiety: $F(1,1276)=47.20, p<0.001, \eta_{p}^{2}=0.04$, depression: $\left.F(1,1276)=117.39, p<0.001, \eta_{p}^{2}=0.08\right)$. The Ménière disease group also reported more health anxiety $(F(1,1276)=53.73$, $\left.p<0.001, \eta_{p}^{2}=0.04\right)$. No difference was found between the Ménière and control group for intolerance of uncertainty. No interaction effects were found between age, gender, and group.

The proportion of each group with clinical levels of anxiety, depression, health anxiety, and PTSD is shown in Table 4. The proportion of the Ménière disease group with clinical levels of distress was significantly higher than the control group for anxiety $\left(\chi^{2}[2, N=\right.$ $800]=253.47, p<0.001)$, depression $\left(\chi^{2}[2, N=800]=859.53\right.$, $p<0.001)$, and health anxiety $\left(\chi^{2}[2, N=800]=145.76, p<\right.$ $0.001)$. Proportions ranged from 1.8 to 9.8 times higher in the Ménière group than the control group.

A total of $16.4 \%$ of the Ménière group met the criteria for PTSD of scores of 44 or more on the PCL, whereas $12.1 \%$ met the criteria of the National Center for PTSD. The presence of only one of the $\mathrm{B}, \mathrm{C}$, or D symptomatic clusters in response to a traumatic

TABLE 4. Clinical Groupings of Ménière's and Control Groups for Anxiety, Depression, Health Anxiety, and PTSD

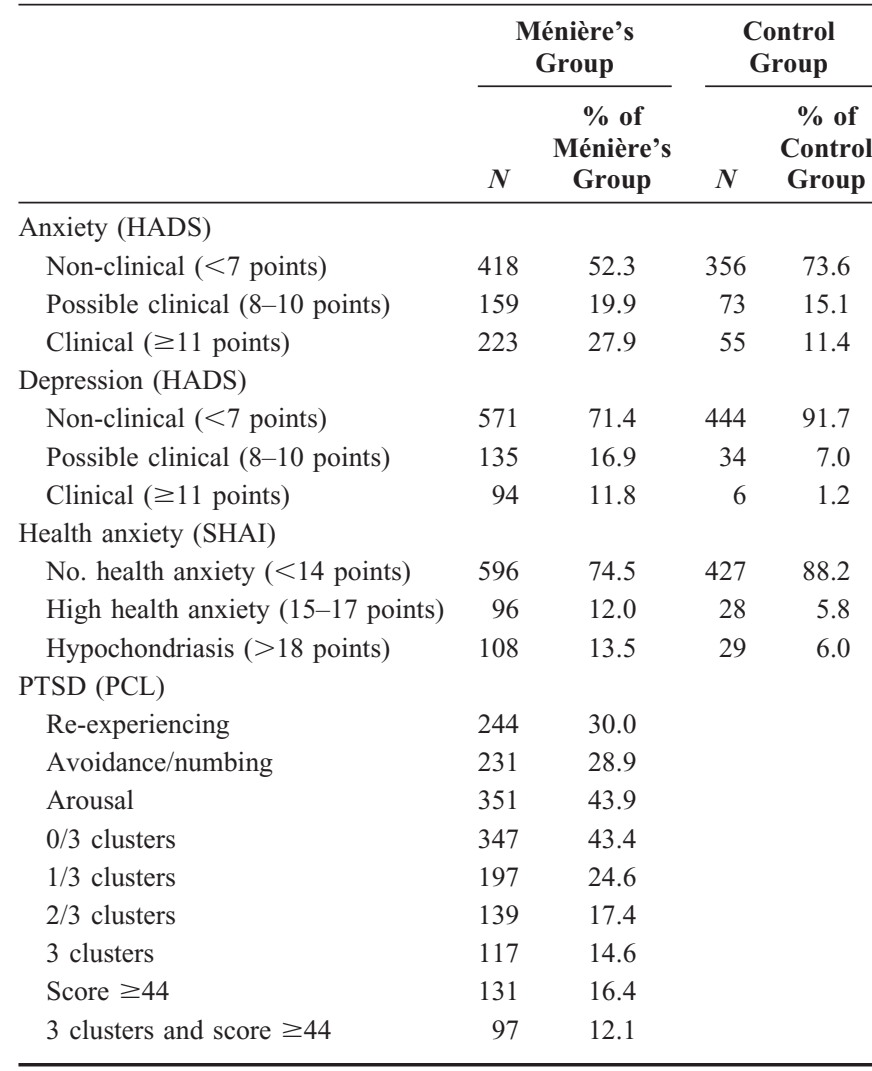


event that is causing distress can be taken as an indication of partial PTSD (Mylle and Maes, 2004). Using this criterion, $42 \%$ of participants reached the threshold for 1 or 2 , but not all 3 , of the symptomatic clusters.

\section{DISCUSSION}

Our findings suggest that the modifiable psychological mechanisms of PTSD, health anxiety, and intolerance of uncertainty do contribute to distress in this group of members of the Ménière Society. The presence and contribution of PTSD symptoms to distress is particularly noteworthy, as the proportion of this sample of people with Ménière disease who met the criteria for PTSD is much higher than the general population level of $1.5 \%$ to $1.8 \%$ (National Institute for Clinical Excellence, 2005). This proportion is also higher than those who have had a stroke $(9.8 \%)$, and is comparable to people who have had a myocardial infarction $(0 \%-16 \%)$ or cardiac surgery (10.8\%-18\%; Tedstone and Tarrier, 2003). According to the criteria for partial PTSD proposed by Mylle and Maes (2004), over half of the sample met criteria for partial or full PTSD.

As was found in this study, the occurrence of PTSD with anxiety and/or depression is common (National Institute for Clinical Excellence, 2005). It has been suggested that PTSD often remains unrecognized when the primary presenting problem is depression or an anxiety disorder (Zimmerman and Mattia, 1999). This may contribute to why PTSD has not been explored as a possible mechanism for distress in people with Ménière disease before. The comorbidity of PTSD with anxiety and/or depression should not complicate treatment of either condition, as the effective treatment of PTSD has been found to improve untreated symptoms of anxiety and depression (National Institute for Clinical Excellence, 2005). It may therefore be beneficial for clinicians to screen patients who seem particularly distressed and poorly adjusted to having Ménière disease for the possible presence of PTSD.

The levels of health anxiety reported by participants with Ménière disease were significantly higher than those of the control group in this study, and appear to be similar to those of medical patients who were attending a general practice clinic, gastroenterology clinic, or an MRI scan (Salkovskis et al., 2002). The levels of health anxiety reported by people with Ménière disease in this study, however, appear to be lower than those reported by people with panic disorder, and much lower than people with hypochondriasis (Salkovskis et al., 2002).

The level of intolerance of uncertainty in the Ménière group appeared to be comparable to the scores of non-anxious people in other research (Buhr and Dugas, 2002; Buhr and Dugas, 2006), and lower than people with anxiety disorders (Holaway et al., 2006; Ladouceur et al., 1999). This suggests that while high levels of intolerance of uncertainty contribute to distress in the Ménière disease group, the distribution of intolerance of uncertainty within the Ménière group as a whole was comparable to normal populations. PTSD and health anxiety were both directly associated with all 3 types of distress. Intolerance of uncertainty, however, was associated with the different types of distress in different ways. A high level of intolerance of uncertainty was directly and quite strongly associated with anxiety, but its effect on depression and handicap was almost fully mediated by PTSD symptoms.

The main strengths of our study were that it is the first to assess the presence and role of PTSD and health anxiety in a large sample of people with Ménière disease. The study is limited, however, by a cross-sectional design using a self-selected sample from a self-help group. It is possible that elevated levels of distress may have been a motivating factor in participants' decision to join the self-help group, and so these results cannot be generalized to all people with Ménière disease without replication in a non self-help group sample. Another limitation of the study is that it was beyond the scope of the study to clinically confirm the diagnosis of Ménière disease in participants, although all participants reported symptoms of vertigo, tinnitus, aural fullness, and hearing disability, and reported that they had been told by a doctor that they have Ménière disease. The recruitment of participants' friends and relatives (without Ménière disease or severe dizziness) as a control group was also a limitation of the study. As this group was a convenience sample and not randomly selected, we cannot assume that the control group is representative of the general population.

The study was also limited by the use of self report measures, although the measure used to assess PTSD in this study (the PCL) has been reported to have good diagnostic efficiency (Blanchard et al. 1996). PTSD, however, has not previously been studied in people with Ménière disease; given the strong association of PTSD with distress found in this study, future research should seek to replicate this finding using clinical interviews. It should also be noted that this study only measured PTSD symptoms experienced over the past month. Given the fluctuating nature of Ménière symptoms, it would be interesting if future studies could assess the lifetime prevalence of PTSD symptoms in people with Ménière disease.

\section{CONCLUSIONS}

Our study has shown that PTSD, health anxiety, and intolerance of uncertainty may be relevant mechanisms for understanding distress among people with Ménière disease. As previous research with other populations has shown that these mechanisms are modifiable with psychological treatment, our study has important implications for the psychological treatment of distress in people with Ménière disease.

\section{REFERENCES}

Alonzo A (2000) The experience of chronic illness and post-traumatic stress disorder: the consequences of cumulative adversity. Soc Sci Med. 50:14751484 .

Andersson G, Asmundson GJ, Denev J, Nilsson J, Larsen HC (2006) A controlled trial of cognitive-behavior therapy combined with vestibular rehabilitation in the treatment of dizziness. Behav Res Ther. 44:1265-1273.

Balaban CD, Thayer JF (2001) Neurological bases for balance-anxiety links J Anxiety Disord. 15:53-79.

Baron RM, Kenny DA (1986) The moderator-mediator variable distinction in social psychological research: conceptual, strategic, and statistical considerations. J Pers Soc Psychol. 51:1173-1192.

Best C, Eckhardt-Henn A, Diener G, Bense S, Breuer P, Dieterich M (2006) Interaction of somatoform and vestibular disorders. J Neurol Neurosurg Psychiatry. 77:658-664.

Blanchard EB, Jones-Alexander J, Buckley TC, Forneris CA (1996) Psychometric properties of the PTSD Checklist (PCL). Behav Res Ther. 34:669-673.

Brandt T (2000) Management of vestibular disorders. J Neurol. 247:491-499.

Buhr K, Dugas MJ (2002) The intolerance of uncertainty scale: Psychometric properties of the English version. Behav Res Ther. 40:931-945.

Buhr K, Dugas MJ (2006) Investigating the construct validity of intolerance of uncertainty and its unique relationship with worry. Anxiety Disord. 20:222-236.

Cass CP (1999) Staging and outcomes for Meniere's disease. In JP Harris (Ed), Meniere disease (pp. 311-325). The Hague, The Netherlands: Kugler Publications.

Cohen H, Ewell LR, Jenkins HA (1995) Disability in Meniere disease. Arch Otolaryngol Head Neck Surg. 121:29-33.

Cramer V, Torgersen S, Kringlen E (2005) Quality of life and anxiety disorders: A population study. J Nerv Ment Dis. 193:196-202.

Dugas MJ, Hedayati M, Karavidas A, Buhr K, Francis K, Phillips NA (2005) Intolerance of uncertainty and information processing: Evidence of biased recall and interpretations. Cognit Ther Res. 29:57-70.

Dugas MJ, Ladouceur R (2000) Treatment of GAD: Targeting intolerance of uncertainty in two types of worry. Behav Modif. 24:635-657.

Elwood S, Carlton JH, Cliffe MJ (1982) A psychological contribution to the management of Meniere disease. Practitioner. 226:1149-1152.

Fava GA, Freyberger HJ, Bech P, Christodoulou G, Sensky T, Theorell T (1995) 
Diagnostic criteria for use in psychosomatic research. Psychother Psychosom. 63:1-8.

Furer P, Walker JR, Freeston MH (2001) Approach to integrated cognitive-behaviour therapy for intense illness worries. In GJG Asmundson, S Taylor, BJ Cox (Eds), Health Anxiety: Clinical and Research Perspectives on Hypochondriasis and Related Conditions (pp. 161-192). Chichester, United Kingdom: Wiley.

Furman JM, Jacob RG (2001) A clinical taxonomy of dizziness and anxiety in the otoneurological setting. Anxiety Disord. 15:9-26.

Hagnebo C, Melin L, Larsen HC (1998) Cognitive behavioural treatment of a patient suffering from Meniere disease. Scand J Behav Ther. 27:42-48.

Holaway RM, Heimberg RG, Coles ME (2006) A comparison of intolerance of uncertainty in analogue obsessive-compulsive disorder and generalized anxiety disorder. Anxiety Disord. 20:158-174.

Jacob RG, Furman JM, Cass SP (2002) Psychiatric consequences of vestibular dysfunction. In Luxon L, Martini A, Furman J, Stephens D (Eds), Audiological Medicine (pp. 869-838). Taylor and Francis.

Jacob RG, Whitney SL, Detweiler-Shostak G, Furman JM (2001) Vestibular rehabilitation for patients with agoraphobia and vestibular dysfunction. A pilot study. Anxiety Disord. 15:131-146.

Jacobson GP, Newman CW (1990) The development of the dizziness handicap inventory. Arch Otolaryngol Head Neck Surg. 116:424-427.

Johansson M, Akerlund D, Larsen HC, Andersson G (2001) Randomized controlled trial of vestibular rehabilitation combined with cognitive-behavioural therapy for dizziness in older people. Otolaryngol Head Neck Surg. 125:151-156.

Jones FA (2002) The role of bibliotherapy in health anxiety: An experimental study. Br J Community Nurs. 7:498-504.

Kangas M, Henry JL, Bryant RA (2002) Posttraumatic stress disorder following cancer: A conceptual and empirical review. Clin Psychol Rev. 22:499-524.

Kirby S, Yardley L (2008) Understanding psychological distress in Meniere disease: A systematic review. Psychol Health Med. 13:257-273.

Kirby S, Yardley L (2009) Cognitions associated with anxiety in Meniere disease. $J$ Psychosom Res. 66:111-118.

Ladouceur R, Dugas MJ, Freeston MH, Rheaume J, Blais F, Boisvert JM (1999) Specificity of generalized anxiety disorder symptoms and processes. Behaviour Therapy, 30:191-207.

Lutman ME, Brown EJ, Coles RR (1987) Self-reported disability and handicap in the population in relation to pure-tone threshold, age, sex, and type of hearing loss. Br J Audiol. 21:45-58.
Mylle J, Maes M (2004) Partial posttraumatic stress disorder revisited. J Affect Disord. 78:37-48.

National Institute for Clinical Excellence (2005) Posttraumatic Stress Disorder: The Management of PTSD in Adults and Children in Primary and Secondary Care. London: Gaskell and the British Psychological Society. National Clinical Practice Guideline Number 26.

Pollak L, Klein C, Rafael SJ, Vera K, Rabey JM (2003) Anxiety in the first attack of vertigo. Otolaryngol Head Neck Surg. 128:829-834.

Rode S, Salkovskis P, Dowd H, Hanna M (2006) Health anxiety levels in chronic pain clinic attenders. J Psychosom Res. 60:155-161.

Salkovskis PM, Rimes KA, Warwick HM, Clark DM (2002) The Health Anxiety Inventory: development and validation of scales for the measurement of health anxiety and hypochondriasis. Psychol Med. 32:843-853.

Stahle J, Arenberg IK, Goldman G (1981) Staging Meniere disease: Description of a vertigo-disability profile. Am J Otol. 2:357-364.

Tedstone JE, Tarrier N (2003) Posttraumatic stress disorder following medical illness and treatment. Clin Psychol Rev. 23:409-448.

Weathers F, Litz B, Herman D, Hiska J, Keane T (1993) The PTSD Checklist (PCL): Reliability, validity and diagnostic utility. Paper presented at: The Annual Meeting of the International Society for Traumatic Stress Studies: San Antonio (TX).

Yardley L (1997) Vertigo and dizziness. In A Baum, S Newman, J Weinman, R West, C McManus (Eds), Cambridge Handbook of Psychology, Health and Medicine (pp. 614-615). Cambridge (MA): Cambridge University Press.

Yardley L, Burgneay J, Nazareth I, Luxon L (1998) Neuro-otological and psychiatric abnormalities in a community sample of people with dizziness: A blind, controlled investigation. J Neurol Neurosurg Psychiatry. 65:679-684.

Yardley L, Dibb B, Osborne G (2003) Factors associated with quality of life in Meniere disease. Clin Otolaryngol. 28:436-441.

Yardley L, Kirby S (2006) Evaluation of Booklet-Based Self-Management of Symptoms in Ménière Disease: A Randomized Controlled Trial. Psychosom Med. 68:762-769.

Yardley L, Masson E, Verschuur C, Haacke N, Luxon L (1992) Symptoms, anxiety and handicap in dizzy patients: Development of the vertigo symptoms scale. J Psychosom Res. 36:731-741.

Zigmond AS, Snaith RP (1983) The hospital anxiety and depression scale. Acta Psychiatr Scand. 67:361-370.

Zimmerman M, Mattia JI (1999) Is posttraumatic stress disorder under diagnosed in routine clinical settings? J Nerv Ment Dis. 187:420-428. 


\section{AUTHOR QUERIES}

\section{AUTHOR PLEASE ANSWER ALL QUERIES}

AQ6 - Please provide the Department/Division for the given affiliation.

AQ1-Please expand BPPV here.

AQ5-Please provide the figure legends in the text.

AQ2-Please check whether the changes made in "F R2 Change $(7,790)$ " is OK.

AQ3-Please provide the location of the publisher of the reference "Jacob RG, Furman JM, Cass SP (2002)"

AQ4 - Please check whether the given year and volume number for reference "Kirby S, Yardley L (2009)" is ok. 